Z. Klin. Chem. Klin. Biochem.

12. Jg. 1974, S. $154-158$

\title{
Enzymdiagnostik bei Patienten mit Hyperlipoproteinämie: Beseitigung von Plasmatrübungen durch selektive Polyanionenpräzipitation von Plasma-Lipoproteinen.
}

\author{
Von J. Lambrecht und D. Seidel \\ Klin.-Chem. Laboratorium der Med. Univ.-Klinik Heidelberg
}

(Eingegangen am 2. Februar 1974)

\begin{abstract}
Es wird über eine einfache Methode berichtet, die es gestattet, Enzymaktivitätsbestimmungen an trüben Plasmen von Patienten mit Hyperlipoproteinämie zuverlässig durchzuführen.

An 30 klaren Plasmen unterschiedlicher Enzymaktivitäten wurden vor und nach Lipoproteinpräzipitation durch Zusetzen eines Heparin- $\mathrm{MgCl}_{2}-\mathrm{Gemischs}$ folgende Enzyme untersucht: Aspartat-Aminotransferase (EC 2.6.1.1), Alanin-Aminotransferase (EC 2.6.1.2), $\gamma$-Glutamyltranspeptidase (EC 2.3.2.1), alkalische Phosphatase (EC 3.1.3.1), Leucinaminopeptidase (EC 3.4.1.1),

Lactatdehydrogenase (EC 1.1.1.27), Creatinkinase (EC 2.7.3.2), saure Phosphatase (EC 3.1.3.2) und $\alpha$-Amylase (EC 3.2.1.1). Nur bei der sauren Phosphatase und der $\alpha$-Amylase kam es zu einer erheblichen inkonstanten Inaktivierung durch die Präzipitation. Alle übrigen Enzymaktivitäten zeigten praktisch keine Veränderungen.

Um in einem Modellversuch den Einfluß hoher Lipoproteinkonzentrationen zu klären, wurden außerdem bei 15 der 30 Plasmen in weiteren Ansätzen gereinigte VLDL $^{1}$ ) zugesetzt. Auch hierbei ergab die Bestimmung aller getesteten Enzymaktivitäten nach Lipoproteinpräzipitation zuverlässige Resultate.
\end{abstract}

\section{A simple method for the determination of enzyme activities in turbid plasma of patients with hyperlipoproteinemia}

This study presents for the first time a simple technique which allows enzyme determinations after the removal of very low density lipoproteins (VLDL $d<1.006 \mathrm{~g} / \mathrm{ml}$ ) from the turbid plasma by precipitation with polyanionic compounds $\left(\mathrm{MgCl}_{2}-\mathrm{Na}-\mathrm{Heparinate}\right.$ mixture).

Using 30 non-turbid plasma samples of different enzy-me activities alanine aminotransferase (2.6.1.2), aspartate aminotransferase (2.6.1.1), $\gamma$-glutamyl transpeptidase (2.3.2.1), lactate dehydrogenase (1.1.1.27), alkaline phosphatase (3:1.3.1), leucine aminopeptidase (3.4.1.1), creatine kinase (2.7.3.2), acid phosphatase (3.1.3.2), and $\alpha$-amylase (3.2.1.1) were determined before and after precipitation with polyanionic compounds. Only in the case of acid phosphatase and $\alpha$-amylase, did the precipitation technique show a significant influence on the enzyme activity, while all other enzymes showed almost identical activities before and after precipitation.

In order to study the effect of high lipoprotein concentrations in the plasma, purified VLDL ${ }^{1}$ ) were added in various amounts to 15 plasma samples of known enzyme activity. Under these conditions also, the determinations of all of the investigated enżyme activities gave reliable results.

Plasmatrübungen sind in der Regel durch erhöhte Konzentrationen von triglyceridreichen Lipoproteinen bedingt. Es handelt sich hierbei um Lipoproteine der Dichteklasse $\mathrm{d}<1,006 \mathrm{~g} / \mathrm{ml}$, die sogenannten ,very low density"-Lipoproteine (VLDL). Bei verschiedenen Formen von primären und sekundären Hyperlipoproteinämien findet sich eine Erhöhung der VLDL im engeren Sinne, die nach ihrer elektrophoretischen Wanderungsgeschwindigkeit auch als prä- $\beta$-Lipoproteine bezeichnet werden. Eine Chylomikronämie kann hinzukommen (Übersicht siehe bei Fredrickson et al (1)). In diesen Fällen können photometrische Untersuchungen, insbesondere Enzymbestimmungen, empfindlich gestört oder ganz unmöglich sein. Das gilt vor allem dann, wenn im kurzwelligen Bereich gemessen wird. Gerade Erkrankungen, die wichtige Domänen der Enzymdiagnostik darstellen, wie z. B. der Herzinfarkt oder Lebererkrankungen, sind häufig mit Hyperlipoproteinämien kombiniert. Es ist daher von besonderem Interesse, auch in solchen Fällen Enzymbestimmungen durchführen zu können.

1) VLDL = Very low density lipoproteins
Bereits 1955 hat Bernfeld (2) Verfahren angegeben, Plasma-Lipoproteine mit Hilfe von Polyanionen selektiv zu präzipieren. Diese Methodik wurde in den folgenden Jahren von Burstein et al (3) weiter ausgebaut. Ziel unserer Untersuchungen war es, eine für VLDL spezifische Präzipitationsmethode zu entwickeln, die es gestattet, auch an trüben Plasmen nach vorheriger Klärung eine Reihe klinisch wichtiger Enzyme einfach und zuverlässig zu bestimmen.

\section{Material und Methodik}

\section{Lösungen}

Heparin

Liquemin-Ampullen, $500 \mathrm{IE} . / \mathrm{ml}$, Hoffmann-La Roche, Grenzach oder Heparin, $50 \mathrm{~g} / 1$, der Fa. Serva, Heidelberg.

$\mathrm{MgCl}_{2}$-Lösung, $2 \mathrm{~mol} / 1$ (Fa. Merck, Darmstadt)

\section{Untersuchungsmaterial}

Jedes Enzym wurde an 30 klaren Plasmen verschiedener Enzymaktivität eines unausgewählten Krankengutes von ambulanten und stationären Patienten der Med. Univ.-KKlinik Heidelberg getestet (Blutentnahme in Ammonium-Heparinat-Röhrchen der Fa. Sarstedt, Nümbrecht). 


\section{Enzymbestimmungen}

Es wurden die folgenden Enzymaktivitäten untersucht:Die $\alpha-$ Amylase wurde mit dem Phadebas-Amylase-Test der Fa. Pharmacia, Uppsala/Schweden und die alkalische Phosphatase mit Merckotest (Fa. Merck, Darmstadt), alle übrigen Enzyme mit Biochemica-Test-Sets der Fa. Boehringer, Mannheim bestimmt. Außer bei der Leucinaminopeptidase erfolgten die Messungen mit „,optimierten“ Testansätzen nach den Empfehlungen der Deutschen Gesellschaft für Klinische Chemie.

Die Messungen der Aspartat- und Alanin-Aminotransferase, Lactatdehydrogenase und alkalischen Phosphatase wurden am Reaction-Rate-Analyser 8600 der Fa. LKB, Lochham bei $25^{\circ} \mathrm{C}$ vorgenommen. Die übrigen Enzyme wurden am Spektrallinienphotometer der Fa. Eppendorf, Hamburg mit Kompensationsschreiber und Küvettenwechselautomatik bestimmt. Die Bewertung der $\alpha$-Amylase erfolgte als Endwertmessung gegen einen Leerwert nach einer 15 -minütigen Inkubation bei $37^{\circ} \mathrm{C}$ im Wasserbad.

\section{Glucose-Bestimmung}

Die Messungen der Plasma-Glucose wurden am Glucose-Analyzer der Fa. Beckman Instruments, München mit Reagenzien der Fa. Boehringer, Mannheim durchgeführt.

\section{Präzipitationstechnik}

1. Dem Plasma wurden $10 \mathrm{Vol} \%$ einer Mischung von gleichen Teilen Heparinlösung und $2 \mathrm{~mol} / 1 \mathrm{MgCl}_{2}$-Lösung zugesetzt.

2. Nach kurzem Durchmischen und 5-minütigem Stehen bei

Raumtemperatur wurde $10 \mathrm{~min}$ bei $3000 \mathrm{U} / \mathrm{min}$ zentrifugiert.

3. Die an der Oberfläche abgesetzten Lipoproteinpräzipitate wurden durch Filtration entfernt.

4. An dem Filtrat wurden die Enzymaktivitäten in üblicher Weise bestimmt. Die gefundene Aktivität wurde mit dem Verdünnungsfaktor 1,1 multipliziert.

\section{VLDL-Präparation}

Zur Gewinnung von VLDL wurden trübe Plasmen (TriglyceridKonzentrationsbereich von $6,85-15,72 \mathrm{~g} / \mathrm{l}$ ) von Blutspendern bei der Dichte $d<1,006 \mathrm{~g} / \mathrm{ml}$ in einer Ultrazentrifuge der $\mathrm{Fa}$. Beckman Instruments, München (Modell Spinco L2 65 B, Rotor Ti 60 ) $24 \mathrm{~h}$ bei $200.000 \mathrm{~g}$ und $4^{\circ} \mathrm{C}$ zentrifugiert. Die im Überstand enthaltenen VLDL wurden durch eine Schneidetechnik gewonnen und ausgiebig (etwa $24 \mathrm{~h}$ bei $4^{\circ} \mathrm{C}$ ) unter mehrfachem Wechsel der Dialyselösung gegen eine Pufferlösung der folgenden Zusammensetzung dialysiert: $1 \mathrm{mmol} / 1 \mathrm{Tris}$, $\mathrm{pH} 7,6 ; 0,15 \mathrm{mmol} / 1$ $\mathrm{NaCl} ; 1,7 \mathrm{mmol} / 1$ EDTA. An der so gewonnenen VLDL-Fraktion wurde der Triglyceridgehalt mit der Methode nach Eggstein und Kreutz (4) (Reagenzein der Fa. Boehringer, Mannheim) bestimmt und die Fraktion durch Einengen (Amicon-Filter) auf einen Triglyceridgehalt von $25 \mathrm{~g} / 1$ eingestellt. Diese VLDL-Suspension wurde dann auf Reinheit bezüglich der zu untersuchenden Enzyme getestet und, sofern keine Enzymaktivität nachweisbar war, als VLDL-Stammlösung im weiteren Untersuchungsgang verwendet.

\section{Behandlung der Plasmen}

Zunächst wurde an jeweils 30 Plasmen die Enzymaktivität vor und nach Heparin- $\mathrm{MgCl}_{2}$-Präzipitation bestimmt.

Bei 15 diescr Plasmen wurden in zwei weiteren Parallelansätzen die präparierten VLDL wie folgt zugesetzt:

VLDL-Ansatz 1: 0,9 ml Plasma + 0,1 ml VLDL-Stammlösung $\widehat{\cong} 2,50 \mathrm{~g} / \mathrm{l}$ zugesetzte Triglyceride im Ansatz. VLDL-Ansatz 2: 0,6 ml Plasma + 0,4 ml VLDL-Stammlösung $\hat{\triangleq} 10,0 \mathrm{~g} / \mathrm{l}$ zugesetzte Triglyceride im Ansatz.

Nach Durchmischen und 60 min Stehenlassen bei Raumtemperatur wurden diese Ansätze weitcrbehandelt wie unter „Präzipitationstechnik" und „Enzymbestimmungen" beschrieben. Bei der Berechnung der Ausgangsaktivität wurden die Verdünnungsfaktoren 11/9 bzw. 11/6 entsprechend berücksichtigt.

\section{Ergebnisse und Diskussion}

Um den Einfluß der Polyanionenpräzipitation auf die verschiedenen Enzymaktivitäten zu untersuchen, wurden 30 klare Plasmen mit der angegebenen Präzipitationstechnik behandelt. Sofort nach Zusetzen des Heparin- $\mathrm{MgCl}_{2}$ Gemischs kam es, bedingt durch die Ausfällung von Lipoproteinen, zu einer homogenen Trübung im Ansatz. Nach unserer Erfahrung reicht 5-minütiges Stehen des Ansatzes nach Zugabe der Präzipitationslösung aus, um auch stark lipämisches Plasma vollständig zu klären. Das Präzipitat wurde wie in der Methodik angegeben eliminiert und die jeweilige Enzymaktivität am klaren Filtrat sowie parallel dazu im unbehandelten Plasma bestimmt. Die statistische Auswertung dieser Ergebnisse ist in Tabelle 1 zusammengefaßt. Bei 7 der 9 untersuchten

\section{Tab. 1. Plasma-Enzymaktivitäten vor und nach Heparin- $\mathrm{MgCl}_{2}-$ Präzipitation}

$\overline{\mathrm{x}}=$ Mittelwerte der Enzymaktivitäten in $\mathrm{U} / \mathrm{l}$ vor Heparin- $\mathrm{MgCl}_{2}-\mathrm{Präzipitation}$

$\overline{\mathbf{y}}=$ Mittelwerte der Enzymaktivitäten in $\mathrm{U} / 1$ nach Haparin- $\mathrm{MgCl}_{2}-$ Präzipitation

$\mathbf{N}=$ Anzahl der untersuchten Plasmen

$r \quad=$ Korrelationskoeffizient

$a ; b=$ Konstanten der Regressionsgeraden $y=a+b x$

\begin{tabular}{|c|c|c|c|c|c|c|c|c|c|}
\hline & $\begin{array}{l}\text { Aspartat- } \\
\text { Amino- } \\
\text { transferase }\end{array}$ & $\begin{array}{l}\text { Alanin- } \\
\text { Amino- } \\
\text { transferase }\end{array}$ & $\begin{array}{l}\gamma \text {-Glutamyl- } \\
\text { trans- } \\
\text { peptidase }\end{array}$ & $\begin{array}{l}\text { alkalische } \\
\text { Phosphatase }\end{array}$ & $\begin{array}{l}\text { Leucin } \\
\text { Amino- } \\
\text { peptidase }\end{array}$ & $\begin{array}{l}\text { Lactat- } \\
\text { dehydro- } \\
\text { genase }\end{array}$ & $\begin{array}{l}\text { Creatin- } \\
\text { kinase }\end{array}$ & $\begin{array}{l}\text { saure } \\
\text { Phos- } \\
\text { phatase }\end{array}$ & $\alpha$-Amylase \\
\hline $\mathrm{N}$ & 30 & 30 & 30 & 30 & 30 & 30 & 30 & \multirow{7}{*}{$\begin{array}{l}\text { inkonstante } \\
\text { Restaktivität } \\
\text { von } 0-50 \% \\
\text { (Trübung im } \\
\text { Testansatz) }\end{array}$} & inkonstante \\
\hline Bereich & {$[7-420]$} & {$[7-500]$} & {$[15-311]$} & [80-938] & {$[8-42]$} & {$[108-880]$} & {$[25-551]$} & & von $0-60 \%$ \\
\hline$\overline{\mathbf{x}}$ & 47,1 & 45,6 & 106,3 & 215,4 & 20,6 & 221,4 & 128,3 & & \\
\hline$\overline{\mathbf{y}}$ & 48,1 & 46,4 & 106,0 & 217,7 & 21,1 & 222,1 & 131,3 & & \\
\hline $\mathbf{r}$ & $+0,999$ & $+0,999$ & $+0,998$ & $+0,999$ & $+0,992$ & $+0,997$ & $+0,999$ & & \\
\hline a & $-0,023$ & $+0,275$ & $-0,406$ & $-1,542$ & $-0,213$ & $-3,318$ & $-0,300$ & & \\
\hline b & $+1,021$ & +1.013 & $+1,001$ & $+1,018$ & $+1,033$ & $+1,018$ & $+1,026$ & & \\
\hline
\end{tabular}

\section{Wilcoxon-}

Test:

$c_{0,05}=14,0$

c $=18,0$

12,0

14,8

$\begin{array}{rr}28,5 & 22,0 \\ 5,5 & 14,0\end{array}$

14,0

35,0

10,0

35,0

$\begin{array}{lllll}5,5 & 14,0 & 0,0 & 10,0 & 51,0\end{array}$


Tab. 2. Plasma-Enzymaktivitäten ohne Behandlung und nach Heparin- $\mathrm{MgCl}_{2}$-Präzipitation ohne und mit VLDL-Zugabe

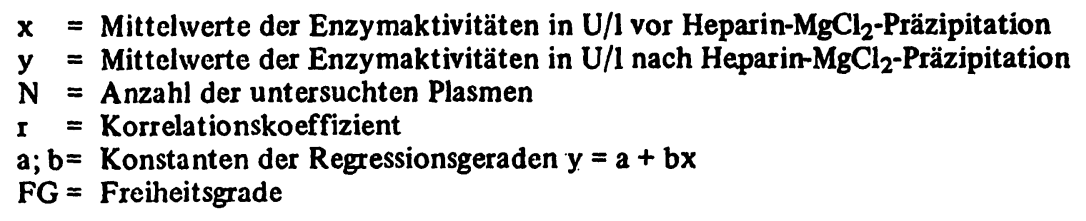

\begin{tabular}{|c|c|c|c|c|c|c|c|}
\hline \multirow[t]{2}{*}{ Enzym } & \multicolumn{2}{|c|}{ ohne Behandlung } & \multicolumn{4}{|c|}{ nach Heparin- $\mathrm{MgCl}_{2}$-Präzipitation } & \multirow{2}{*}{$\begin{array}{l}\text { Friedman-Test } \\
F G=3 ; x_{0.05}^{2} \\
=7,815 \\
x^{2}\end{array}$} \\
\hline & & & & ohne VLDL & $\begin{array}{l}\text { nach Zugabe } \\
\text { von VLDL } 1 \\
\triangleq 2,50 \mathrm{~g} / 1 \\
\text { Triglyceride }\end{array}$ & $\begin{array}{l}\text { nach Zugabe } \\
\text { von VLDL } 2 \\
\text { A } 10,0 \mathrm{~g} / 1 \\
\text { Triglyceride }\end{array}$ & \\
\hline \multirow[t]{2}{*}{ Aspartat-Aminotransferase } & $\overline{\mathbf{x}}$ & $36 ; 5$ & $\overline{\mathbf{y}}$ & 37,1 & 38,7 & 39,6 & $18,86^{\circ}$ \\
\hline & $\begin{array}{l}\mathrm{N} \\
\mathrm{Be}- \\
\text { reich }\end{array}$ & $\begin{array}{l}15 \\
{[8-166]}\end{array}$ & $\begin{array}{l}\mathbf{r} \\
\mathbf{a} \\
\mathbf{b}\end{array}$ & $\begin{array}{l}+0,999 \\
-0,057 \\
+1,018\end{array}$ & $\begin{array}{l}+0,999 \\
+0,257 \\
+1.024\end{array}$ & $\begin{array}{l}+0,999 \\
+1,373 \\
+1,019\end{array}$ & \\
\hline \multirow[t]{2}{*}{ Alanin-Aminotransferase } & $\overline{\mathbf{x}}$ & 31,9 & $\overline{\mathbf{y}}$ & 32,6 & 32,9 & 33,8 & 17,90 \\
\hline & $\begin{array}{l}\mathrm{N} \\
\mathrm{Be}- \\
\text { reich }\end{array}$ & $\begin{array}{l}15 \\
{[10-160]}\end{array}$ & $\begin{array}{l}\mathbf{r} \\
\mathbf{a} \\
\mathrm{b}\end{array}$ & $\begin{array}{l}+0,999 \\
+0,093 \\
+1,018\end{array}$ & $\begin{array}{r}+0,999 \\
+0,138 \\
1,027\end{array}$ & $\begin{array}{r}+0,999 \\
+1,037 \\
1,026\end{array}$ & \\
\hline \multirow[t]{2}{*}{$\gamma$-Glutamyltranspeptidase } & $\overline{\mathbf{x}}$ & 98,5 & $\overline{\mathbf{y}}$ & 98,0 & 99,4 & 100,5 & 6,60 \\
\hline & $\begin{array}{l}\mathrm{N} \\
\mathrm{Be} \\
\text { reich }\end{array}$ & $\begin{array}{l}15 \\
{[15-231]}\end{array}$ & $\begin{array}{l}\mathbf{r} \\
\mathbf{a} \\
\mathrm{b}\end{array}$ & $\begin{array}{l}+0,997 \\
-0,170 \\
+0,997\end{array}$ & $\begin{array}{l}+0,998 \\
-0,641 \\
+1,016\end{array}$ & $\begin{array}{l}+0,998 \\
+1,311 \\
+1,007\end{array}$ & \\
\hline \multirow[t]{2}{*}{ Alkalische Phosphatase } & $\overline{\mathbf{x}}$ & 279,9 & $\overline{\mathbf{y}}$ & 283,7 & 286,5 & 288,9 & 10,98 \\
\hline & $\begin{array}{l}\mathrm{N} \\
\mathrm{Be} \\
\text { reich }\end{array}$ & $\begin{array}{l}15 \\
{[90-938]}\end{array}$ & $\begin{array}{l}\mathbf{r} \\
\mathbf{a} \\
\mathbf{b}\end{array}$ & $\begin{array}{l}+0,999 \\
-1,450 \\
+1.019\end{array}$ & $\begin{array}{l}+0,999 \\
+6,041 \\
+1,002\end{array}$ & $\begin{array}{l}+0,998 \\
+9,230 \\
+0,999\end{array}$ & \\
\hline \multirow[t]{2}{*}{ Leucin Aminopeptidase } & $\overline{\mathbf{x}}$ & 24,1 & $\overline{\mathbf{y}}$ & 24,9 & 25,5 & 25,5 & 13,58 \\
\hline & $\begin{array}{l}\mathrm{N} \\
\mathrm{Be}- \\
\text { reich }\end{array}$ & $\begin{array}{l}15 \\
{[13-42]}\end{array}$ & $\begin{array}{l}\mathbf{r} \\
\mathbf{a} \\
\mathbf{b}\end{array}$ & $\begin{array}{l}+0,991 \\
+0,001 \\
+1,036\end{array}$ & $\begin{array}{l}+0,983 \\
+1,155 \\
+1,013\end{array}$ & $\begin{array}{l}+0,981 \\
+1,304 \\
+0,986\end{array}$ & \\
\hline \multirow[t]{2}{*}{ Lactatdehydrogenase } & $\overline{\mathbf{x}}$ & 180.3 & $\overline{\mathbf{y}}$ & 183,3 & 185,7 & 190,7 & 25,46 \\
\hline & $\begin{array}{l}\mathrm{N} \\
\mathrm{Be}- \\
\text { reich }\end{array}$ & $\begin{array}{c}15 \\
{[108-367]}\end{array}$ & $\begin{array}{l}\mathbf{r} \\
\mathbf{a} \\
\mathbf{b}\end{array}$ & $\begin{array}{l}+0,997 \\
-3,669 \\
+1,037\end{array}$ & $\begin{array}{l}+0,998 \\
-4,875 \\
+1,057\end{array}$ & $\begin{array}{l}+0,996 \\
-0,055 \\
+1,058\end{array}$ & \\
\hline \multirow[t]{2}{*}{ Creatinkinase } & $\overline{\mathbf{x}}$ & 112,1 & $\overline{\mathbf{y}}$ & 113,2 & 112,5 & 116,7 & 8,46 \\
\hline & $\begin{array}{l}\mathrm{N} \\
\mathrm{Be}- \\
\text { reich }\end{array}$ & $\begin{array}{l}15 \\
{[31-254]}\end{array}$ & $\begin{array}{l}r \\
a \\
b\end{array}$ & $\begin{array}{l}+0,999 \\
+2,094 \\
+0,995\end{array}$ & $\begin{array}{l}+0,998 \\
+2,307 \\
+0,983\end{array}$ & $\begin{array}{l}+0,996 \\
+2,627 \\
+1,017\end{array}$ & \\
\hline
\end{tabular}

Enzyme ergab sich eine hochgradige Ubereinstimmung der Meßwerte vor und nach Präzipitation. Der Steigungsfaktor $b$ der Regressionsgeraden liegt in allen diesen Fällen geringgradig über $+1, d . h$., es ist ein geringfügiger Trend zum Anstieg der gemessenen Enzymaktivitäten nach Polyanionenpräzipitation zu verzeichnen. Dieser Trend war im Wilcoxon-Test an verbundenen Stichproben aber lediglich bei der Aspartat- und Alanin-Aminotransferase sowie Creatinkinase signifikant. Als Signifikanzniveau wurde bei diesem wie auch beim folgenden Friedman-Test a $=0,05$ festgelegt, da bei der Art der Fragestellung (Aufrechterhaltung der Nullhypothese) ein großer Fehler 1. Art zugelassen werden mußte. Die Korrelationskoeffizienten waren bei diesen 7 Enzymen ausnahmslos größer als + 0,99. Es liegt somit bei diesen Enzymen eine hervorragende Korrelation der Aktivitätswerte vor und nach Lipoproteinpräzipitation vor.

Bei der Bestimmung der sauren Phosphatose an den Filtraten nach Heparin- $\mathrm{MgCl}_{2}$-Präzipitation kam es nach Zusetzen der Plasma-Präparation zum Puffer-SubstratGemisch zur massiven Trübung im Testansatz, wahrscheinlich bedingt durch Ausfällung des $p$-Nitrophenylphosphates. Es konnte lediglich eine inkonstante Restaktivität von 0-50\% der Ausgangsaktivität gefunden wer- 
Tab. 3. Plasma-Glucose-Konzentrationen ohne Behandlung und nach Heparin- $\mathbf{M g C l}_{\mathbf{2}}-\mathrm{Präzipitation} \mathrm{ohne} \mathrm{und} \mathrm{mit} \mathrm{VLDL-Zugabe}$

$\overline{\mathbf{x}}=$ Mittelwert der Glucose-Konzentrationen in $\mathrm{mg} / \mathbf{l}$ vor Heparin- $\mathrm{MgCl}_{2}$-Präzipitation

$\bar{y}=$ Mittelwert der Glucose-Konzentrationen in $\mathrm{mg} / 1$ nach Heparin- $\mathrm{MgCl}_{2}-$ Präzipitation

$\mathbf{N}=$ Anzahl der untersuchten Plasmen

$r=$ Korrelationskoeffizient

$a ; b=$ Konstanten der Regressionsgeraden $y=a+b x$

FG $=$ Freiheitsgrade

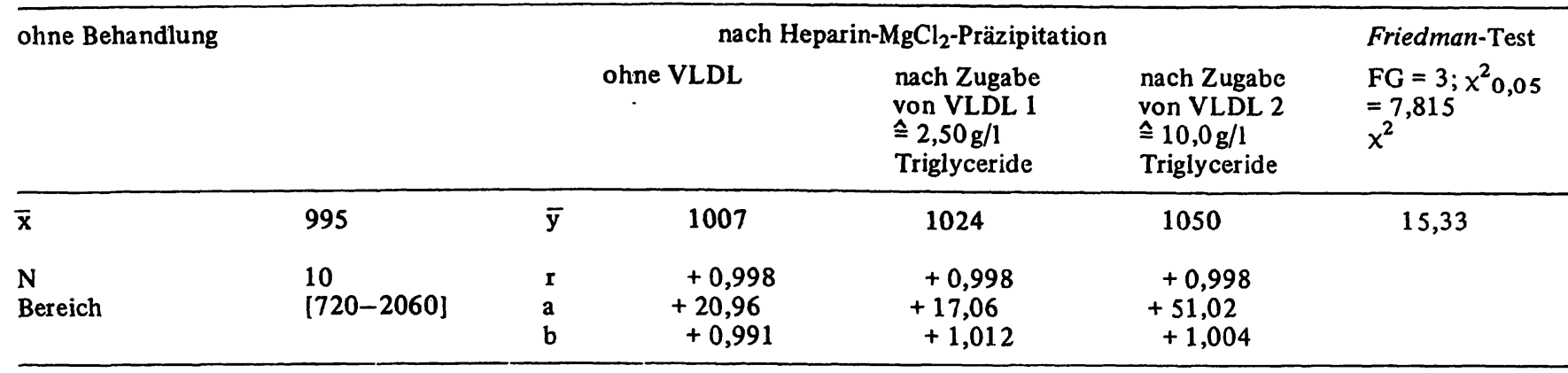

den. Diese Schwierigkeit trat bei der Bestimmung der alkalischen Phosphatase nicht auf. Bei der $\alpha$-Amylase zeigte sich ebenfalls eine inkonstante Inaktivierung mit Restaktivitäten zwischen 0 und 60\%. Diese beiden Enzym. tests schieden deshalb von der weiteren Untersuchung aus.

Um in einem Modellversuch den Einfluß hoher Lipoproteinkonzentrationen zu klären, wurden bei 15 der oben genannten Plasmen in zwei weiteren Parallalansätzen präparierte Lipoproteine (VLDL) in zwei verschiedenen Konzentrationen zugesetzt. (s. „Behandlung der Plasmen“ unter Methodik).

Im VLDL-Ansatz 1 kam es nach Hinzufügen der VLDLPräparation zu einer deutlichen opaleszenten Trübung des Plasmas, während im VLDL-Ansatz 2 eine massiv milchige Trübung durch VLDL-Zugabe erhalten wurde.

Vor Durchführung der Präzipitation wurde das PlasmaVLDL-Gemisch 1 Stunde bei Raumtemperatur belassen, um etwaige kurzfristige Wechselwirkungen zwischen Plasma und Lipoproteinpräparation abklingen zu lassen. Auch in den VLDL-Ansätzen 2 (s. Methodik) mit VLDLKonzentrationen entsprechend einer Triglyceridkonzentration von über $10.0 \mathrm{~g} / 1$ war in allen Fällen mit der angegebenen Präzipitationstechnik eine einwandfreie Klärung des Plasmas zu erzielen. Orientierende Untersuchungen an Nativplasmen von Patienten mit Hyperlipoproteinämie Typ IV und V mit Triglyceridwerten zwischen $\mathbf{3 0}$ und $110 \mathrm{~g} / \mathrm{l}$ zeigten, daß dies auch für extrem hohe VLDL-Konzentrationen zutrifft. Aus technischen Gründen war es allerdings nicht möglich, den Modellversuch mit so hohen VLDL-Konzentrationen durchzuführen.

Die Ergebnisse des VLDL-Zusatzversuchs sind in Tabelle 2 dargestellt. Die gute Úbereinstimmung der Mittelwerte und die äußerst hohe Korrelation bei allen getesteten Enzymaktivitäten zeigen, daß es auch bei sehr hohen Lipoproteinkonzentrationen im Plasma nicht zu einer Beeinträchtigung dieser Enzymaktivitätsbestimmungen kommt.
Die genaue statistische Analyse zeigt auch hier eine Tendenz zum Aktivitätsanstieg nach der Lipoproteinpräzipitation, die offensichtlich mit steigenden VLDL-Konzentrationen zunimmt. Zur statistischen Überprüfung dieses Sachverhalts bietet sich der Friedman-Test an, da vier verbundene Stichproben vorliegen. Außer bei der $\gamma$-Glutamyltranspeptidase ergibt sich in allen Fällen eine deutliche statistische Signifikanz des Enzymaktivitätsanstiegs in Abhängigkeit von der artefiziell erhöhten VLDL-Konzentration. Jedoch auch nach Zusetzen einer sehr hohen VLDL-Menge entsprechend einer Triglyceridendkonzentration im Plasma von über $10,0 \mathrm{~g} / 1$ übersteigen die gefundenen Meßwerte die Ausgangswerte im Nativplasma im Mittel um nicht mehr als maximal 2-6\%. Um abzuklären, inwieweit dieser Trend durch einen direkten physikochemischen Einfluß der Präzipitationstechnik auf die Enzymaktivitäten als solche bedingt sein könnte, wurde an 10 weiteren Plasmen der VLDL-Zusatzversuch durchgefuhrt und anstelle der Enzymaktivitäten die Plasmaglucose bestimmt. In der hierzu verwendeten VLDL-Suspension selbst war keine Glucose nachweisbar. Wie aus der Tabelle 3 ersichtlich ist, tritt auch hier der gleiche Meßwertanstieg nach Präzipitation in Abhängigkeit von der VLDL-Konzentration im Ansatz auf. Auch dieser Trend war statistisch signifikant. Es bieten sich unseres Erachtens hierfür zwei Erklärungsmöglichkeiten an.

1. Es wäre denkbar, daß die Lipoproteinpräzipitation bedingt durch das relativ große Eigenvolumen der VLDL-Partikel eine Einengung der Plasmaprobe bewirkt.

2. Weiterhin muß die Möglichkeit in Betracht gezogen werden, daß trotz vorheriger Testung der VLDL-Suspension auf Reinheit bezüglich der getesteten Enzyme geringe Enzymmengen durch die VLDL-Zugabe in die Testansätze mit eingebracht worden sind. Dies müßte dann ebenso für die Plasma-Glucose angenommen werden. 
Unabhängig von diesen Fragen ist jedoch ein so geringfügiger Meßwertanstieg von maximal 2-6\% der Gesamtaktivität für klinische Belange ohne Bedeutung.

Zusammenfassend kann gesagt werden, daß die spezifische Lipoproteinpräzipitation eine einfache Möglichkeit bietet, auch in trüben Plasmen hyperlipämischer Patienten ein breites Spektrum klinisch wichtiger Enzyme zuverlässig zu diagnostizieren. Es gab bisher fuir dieses Problem keine befriedigenden Lösungen. Versuche einer Korrektur durch Analysenleerwerte oder Lipidextraktionsverfahren (Richterich (5)) kommen bei Enzymbestimmungen nicht in Betracht. Eine Beseitigung starker Serumtrübungen wurde durch Zentrifugation in Kälte ( $F$. W. Schmidt, persönl. Mitt.) versucht. Aber auch dieses Verfahren ist unbefriedigend, da sicherlich der größte Anteil der VLDL-Fraktion durch diese Maßnahme nicht entfernt werden kann. Auch die Aktivierung der Lipoproteinlipase durch die intravenöse Heparingabe vor der Blutentnahme zur Klärung des Plasmas (Kattermann, persönl. Mitt:) ist nach unserer Erfahrung nicht immer erfolgreich und außerdem umständlich. Das hier geschilderte Verfahren wurde in einem Modellversuch erprobt. Praktische Erfahrungen in dèr klinischen Routinediagnostik müssen noch gewonnen werden!

\section{Danksagung}

Herrn Dr. H. Scheurlen, Abteilung für Medizinische Dokumentation und Statistik der Universität, 69 Heidelberg, Voßstr. 3, danken wir fie die Durchführung des Wilcoxon- und FriedmanTests.

Die Arbeit wurde durch Mittel der Deutschen Forschungsgemeinschaft (SFB 90) unterstützt.

\title{
Literatur:
}

1. Fredrickson, D. S. \& Levy, R. I. (1972) in „The Metabolic Basis of Inherited Disease" (Stanbury, J. B., Wyngaarden, J. B., Fredrickson, D. S. Hrsg.) Third Edition, Mc. Graw-Hill Book Company, London, 545-614.

2. Bernfeld, P. (1955), Fed. Proc. 14, 182.

3. Burstein, M., Scholnik, H. R. \& Morfin, R. (1970), J. Lipid Res. 11, 583-595.

4. Eggstein, M. \& Kreutz, F. H. (1966), Klin. Wochenschr. 44, 262-267.

5. Richterich, R. (1968), „Klinische Chemie“, 2. Aufl., Verlag S. Karger Basel, New York, 65.

\author{
Dr. J. Lambrecht \\ Zentrallabor des \\ Städt. Krankenhauses Kemperhof \\ 5400 Koblenz 1 \\ Koblenzer Str. \\ Prof. Dr. D. Seidel \\ Klin. Chem. Laboratorium \\ der Med. Univ.-Klinik \\ 69 Heidelberg \\ Bergheimer Str. 58
}

